\title{
The Cost of Transplant Immunosuppressant Therapy: Is This Sustainable?
}

\author{
Alexandra James ${ }^{1} \cdot$ Roslyn B. Mannon ${ }^{2}$
}

Published online: 28 March 2015

(C) Springer International Publishing AG 2015

\begin{abstract}
A solid organ transplant is a life-saving therapy that engenders the use of immunosuppressive medications for the lifetime of the transplanted organ and its recipient. Conventional therapy includes both induction therapy (a biologic that is infused perioperatively) followed by maintenance therapy. The cost of these medications is a constant concern, and the advent of generics has brought this cost down modestly. For those lacking long-term insurance coverage, this may be a significant out-of-pocket expense that is not affordable. Moreover, transplant centers are managing higher risk transplant recipients that require more complex induction regimens and longer term use of such biologic agents in the context of desensitization or abrogation of de novo antibody-mediated injury. While in kidney transplantation Medicare part B covers 3 years of medication, there is frequent non-adherence due to cost after that time-point. The impact of the Affordable Care Act remains uncertain at this time. Finally, the pipeline of new therapies is limited due to the cost of development of a drug, the inherent cost of clinical studies, and lack of defined endpoints for newer therapies in high-risk patients. These new therapies are of high value to the community but will contribute additional burden to current drug costs.
\end{abstract}

This article is part of the Topical Collection on Kidney Transplantation

Roslyn B. Mannon

rmannon@uab.edu

1 Department of Pharmacy, University of Alabama at Birmingham, Birmingham, AL, USA

2 Division of Nephrology, Department of Medicine, University of Alabama at Birmingham, 1900 University Boulevard, THT 643, Birmingham, AL 35294, USA
Keywords Immunosuppression $\cdot$ Cost $\cdot$ Outcome $\cdot$ Kidney · Transplantation

\section{Introduction}

Immunosuppression is required for the lifetime of a solid organ transplant to prevent rejection. Therapy begins at the time of transplant using induction therapy. Historically, this consisted of high-dose intravenous corticosteroids, but now entails the use of biological therapies that suppress $\mathrm{T}$ cell function or cause $\mathrm{T}$ cell depletion. These biologics are potent and only used for specific total doses and in the short time period postoperatively. Long-term suppression of the immune response requires a combination of agents taken orally. These typically consist of corticosteroids, a calcineurin inhibitor (CNI), and an anti-metabolite, often mycophenolic acid. Therapy is needed indefinitely, for the duration of the allograft. These agents are specific to mitigating $T$ cell responses against the allograft. When antibody-mediated injury occurs, therapy to mitigate $\mathrm{B}$ cell responses and plasma cells are engaged. To date, there are no FDA-approved medications for antibodymediated rejection (AMR), and so off-label use of biologics and other small molecules becomes commonplace. The advent of these biologics, often adapted from the use in autoimmune disease, has further complicated the cost of therapy.

The average reported cost of a solid organ transplant ranges from $\$ 260,000.00$ for a single kidney transplant to over $\$ 1.2$ million dollars for combined heart and lung transplants [1]. There is a clear cost savings to transplantation in support of kidney failure in place of hemodialysis [2]. However, longterm oral maintenance immunosuppression and other prescription medications can cost patients upwards of $\$ 2500.00$ per month depending on various factors including the number of prescription medications and insurance coverage, with the 
average annual cost of medications in the USA reported between $\$ 10,000$ and $\$ 14,000$ per patient [3]. The documented cost as billed charges for all outpatient drugs prescribed from discharge for the transplant admission to 180 days posttransplant discharge is between $\$ 18,200.00$ and $\$ 30$, 300.00 for kidney transplant and heart transplant, respectively, and higher cost was seen if multiple organs were transplanted [1]. This cost includes immunosuppressant medications and other transplant-related and non-transplant-related prescription medications. It can be a financial burden for patients following transplantation to afford oral maintenance immunosuppression especially those without adequate insurance coverage [4]. Moreover, one should take into consideration the cost and consequences of medication non-adherence [5]. The introduction to the market of a number of generic formulations (mycophenolate mofetil (2008), tacrolimus (2009), mycophenolic sodium and sirolimus (both in 2014)) has and will potentially continue to ease the financial burden; however, conversion concerns exist and even speculate increased initial costs in the short term due to laboratory monitoring [6••].

In this review, we will provide a perspective about the cost of immunosuppression, evaluating each therapy individually, with a focus on kidney transplantation, the most common solid organ transplanted. The perspectives of the patient/recipient, the transplant center, and the payor will be noted, and cost of each agent discussed is summarized in Table 1. Despite changes in the healthcare field in terms of compensation, the growing cost of therapies, and off-label usage, continues to expand. The cost of drug development coupled by the relatively small market of transplantation (a rare disease) is leading to escalating costs born onto the field that are not sustainable in the long term.

\section{Induction Agents}

\section{T Cell Non-depletional: Basiliximab}

Basiliximab (Simulect ${ }^{\circledR}$ ), a monoclonal antibody, acts by binding and blocking the interleukin-2 receptor $\alpha$ chain (IL$2 \mathrm{R} \alpha$; CD25), located on the surface of activated T lymphocytes. Another product, daclizumab, acted in similar fashion but is no longer on the market. Basiliximab is indicated for the prophylaxis of acute organ rejection in patients receiving renal transplantation and is provided as a 20-mg dose intravenously given perioperatively and a second dose on postoperative day 4 [7]. Numerous studies have compared anti-IL2R $\alpha$ induction versus no induction, as well as anti-IL $2 \mathrm{R} \alpha$ induction versus rabbit thymocyte globulin (rATG), a depletional induction therapy discussed below. Compared to no induction therapy (i.e., corticosteroids alone), anti-IL2R $\alpha$ induction reduced the risk of graft loss or death with a functioning transplant, acute rejection, and early malignancy, but did not improve patient survival [8]. While compared to rATG anti-IL2R $\alpha$ resulted in fewer side effects, as well as less CMV disease and malignancy, biopsy-proven acute rejection at 1 year was significantly more common (eight studies: RR $1.3095 \%$ CI 1.01 to 1.67). Finally, in a cost comparison between basiliximab versus placebo (including steroid therapy), no significant differences in costs were seen in terms of immunosuppressive therapies, total hospitalization, laboratory tests, outpatient visits, postoperative dialysis, or total costs at 6 or 12 months from an institutional perspective [9]. The cost, per the average wholesale price (AWP) is $\$ 3245$ per dose with an estimated cost/person of $\$ 6490$ (Table 1).

\section{T Cell Depletion: Rabbit Anti-thymocyte Globulin and Alemtuzumab}

rATG (Thymoglobulin ${ }^{\circledR}$ ) is a polyclonal antibody obtained by the immunization of rabbits with human thymocytes [10] and is thought to induce immunosuppression by $\mathrm{T}$ cell clearance from the circulation and modulation of T cell activation. While rATG is FDA approved for the treatment of steroid-resistant acute rejection [10], nearly $63 \%$ of US transplant centers use it for prophylaxis of acute rejection as induction therapy [11]. While an alternative product, equine anti-thymocyte globulin $\left(\mathrm{ATGAM}^{\circledR}\right)$, is available, rATG is considerably cheaper with cost savings of $\$ 5977$ for standard treatment of acute rejection. These savings were attributed to treatment of fewer recurrent rejection episodes and less frequent dialysis treatment [12]. When compared to no induction, a 5-day course of rATG effectively reduced 1 -year acute rejection rates without significant increase in total inpatient costs or posttransplant complications at one institution [13]. Moreover, rATG induction was associated with higher inpatient costs, considerably from drug cost. As expected, pharmacy cost per patient was also significantly higher in rATG induction group ( $\$ 6810$ versus $\$ 1528$, $p<0.001$ ) [13]. However, total hospitalization costs during the first 12 months after transplantation were comparable between the groups. Although not significant, reported mean readmission cost per patient tended to be lower with rATG than no induction, $\$ 9461$ versus $\$ 15,410$, respectively [13].

Strategies to help minimize cost of this expensive agent include dosing of rATG guided by $\mathrm{CD}^{+} \mathrm{T}$ cell counts as well as delayed administration of doses [14, 15]. For example, intermittent dosing based on $\mathrm{CD}^{+}$count compared to full treatment course (up to 10 daily doses) resulted in $68 \%$ cost savings compared with an equivalent dose of rATG for 10 days and a $46 \%$ cost savings compared with daily administration for 6 days [15]. In this single-center prospective study of 41 kidney transplant recipients, the mean individual rATG dose was $104 \mathrm{mg}$ per subject with an average of 3 doses for a total cumulative of $318 \mathrm{mg}$ per patient, compared to 6-10 in the full treatment group. In another approach, 60 recipients of a deceased-donor kidney transplant were studied 
Table 1 Immunosuppressant medication AWP and average cost per set period of time by dose

\begin{tabular}{|c|c|c|c|c|}
\hline Drug (generic name) & Dosage form & Average dose & AWP (\$) & Estimated cost/month (\$) \\
\hline Prograf (tacrolimus) & 1-mg capsule & $4 \mathrm{mg}$ BID & 5.22 & 1252.80 \\
\hline Tacrolimus & 1-mg capsule & $4 \mathrm{mg}$ BID & 4.45 & 1068.00 \\
\hline Neoral (cyclosporine, modified) & $\begin{array}{l}100 \text {-mg capsule } \\
25 \text {-mg capsule }\end{array}$ & $150 \mathrm{mg}$ BID & $\begin{array}{l}7.79 \\
1.95\end{array}$ & 701.40 \\
\hline Cyclosporine, modified & $\begin{array}{l}100 \text {-mg capsule } \\
\text { 25-mg capsule }\end{array}$ & $150 \mathrm{mg}$ BID & $\begin{array}{l}5.49 \\
1.37\end{array}$ & 493.80 \\
\hline CellCept (mycophenolate) & 250-mg capsule & $1 \mathrm{~g} \mathrm{BID}$ & 7.86 & 1886.40 \\
\hline Mycophenolate & 250-mg capsule & $1 \mathrm{~g} \mathrm{BID}$ & 3.96 & 950.40 \\
\hline Myfortic (mycophenolic acid) & 180-mg tablet & $720 \mathrm{mg}$ BID & 5.08 & 1219.20 \\
\hline Mycophenolic acid & 180-mg tablet & $720 \mathrm{mg}$ BID & 4.56 & 1094.40 \\
\hline Rapamune (sirolimus) & 1-mg tablet & $2 \mathrm{mg}$ daily & 20.20 & 1212.00 \\
\hline Sirolimus & $0.5-\mathrm{mg}$ tablet & $2 \mathrm{mg}$ daily & 8.30 & 1038.00 \\
\hline Zortress (everolimus) & 0.5 -mg tablet & $1 \mathrm{mg}$ BID & 15.90 & 1908.00 \\
\hline Biologic/injectable & Dosage form & Average dose & $\operatorname{AWP}(\$)$ & Estimated cost $/$ dose $(\$)^{\mathrm{a}}$ \\
\hline Simulect (basiliximab) & 20-mg vial & $20 \mathrm{mg}$ & 3244.57 & 3244.57 \\
\hline Thymoglobulin (anti-thymocyte globulin rabbit) & 25-mg vial & $1.5 \mathrm{mg} / \mathrm{kg}$ & 797.35 & 3189.40 \\
\hline Campath (alemtuzumab) & 30-mg vial & $30 \mathrm{mg}$ & NA & NA \\
\hline Nulojix (belatacept) & 250-mg vial & $\begin{array}{l}\text { Induction }(10 \mathrm{mg} / \mathrm{kg}) \\
\text { Maintenance }(5 \mathrm{mg} / \mathrm{kg})\end{array}$ & 1107.60 & $\begin{array}{l}3322.80 \\
2215.20\end{array}$ \\
\hline Soliris (eculizumab) & $10 \mathrm{mg} / \mathrm{mL}(30-\mathrm{mL}$ vial $)$ & $900 \mathrm{mg}$ & 7196.40 & 21589.20 \\
\hline Privigen (immune globulin) & $10 \mathrm{~g}(100 \mathrm{~mL})$ & $1 \mathrm{~g} / \mathrm{kg}$ & 1500.00 & 10500.00 \\
\hline Rituximab (Rituxan) & $10 \mathrm{mg} / \mathrm{mL}(10-\mathrm{mL}$ vial $)$ & $375 \mathrm{mg} / \mathrm{m}^{2}$ & 845.95 & 3383.80 \\
\hline
\end{tabular}

$A W P$ actual wholesale price

${ }^{\text {a }}$ Weight-based dosing assumes a 70-kg patient

retrospectively, in which 28 received short-course rATG consisting of $3-5$ doses of $1.5 \mathrm{mg} / \mathrm{kg}$ and 32 received standard treatment with rATG consisting of 5 daily doses of $1.5 \mathrm{mg} / \mathrm{kg}$ [14]. Patients receiving short-course treatment received a mean total induction dose of $371.8 \pm 124.9 \mathrm{~kg}$ while those in the standard course received a mean total induction dose of $523.4 \pm 115.5 \mathrm{~kg}$. With short-course treatment, there was a $30 \%$ cumulative dose reduction compared to standard treatment and no differences were reported in biopsy-confirmed acute rejection or serum creatinine levels at 3 or 6 months posttransplant. Another cost-saving measure was evaluated by McGillicuddy et al. [16]. They reported that delayed administration of final dose of rATG by administration in the outpatient setting on the day of discharge versus inpatient administration resulted in cost savings of $\$ 860$ per patient without affecting acute rejection rates or readmission rates. This could also result in a mean increase in revenue generation of approximately $\$ 1856$ per patient because the dose was administered in the institutions' outpatient clinic. Interestingly, among patients at high risk for acute rejection (AR) or delayed graft function (DGF), 5-day induction with rATG vs. basiliximab has reduced the incidence 15.6 vs. $25.5 \%(p=$ $0.02)$ and severity of acute rejection 1.4 vs. $8.0 \%(p=0.005)$ but not incidence of DGF. In and of itself, this may reduce cost further avoiding additional procedures and hospitalizations for management of AR.

Alemtuzumab (Campath $\left.{ }^{\circledR}\right)$ is a humanized monoclonal antibody binding to CD52 on the surface of B and T lymphocytes that functions by inducing cellular-mediated lysis following surface binding. This drug is currently FDA approved for treatment of B cell chronic lymphocytic leukemia [17] and has the convenience of requiring only one dose for induction when compared to other available induction agents for kidney transplant recipients. It is characterized by a potent lymphocyte and monocyte depletion, the former sustained for about 6 months. In a large randomized study of kidney transplant recipients consisting of 474 patients from 30 transplant centers, induction with a single $30-\mathrm{mg}$ dose of alemtuzumab was associated with less frequent biopsy-confirmed acute rejection at 1 year (5\%) compared to basiliximab treatment (17\%) in low immunological risk subjects $[18 \bullet \bullet]$. Compared to rATG in high-risk patients, the authors showed similar efficacy when evaluating biopsy-confirmed acute rejection at 6 and 12 months. In 2013, the manufacturer changed their distribution model for alemtuzumab and it is no longer commercially available. It is now provided only through the Campath ${ }^{\circledR}$ Distribution Program free of charge for deemed appropriate patients. While this is an economical advantage to centers at the 
present time, concern remains for the future and availability of alemtuzumab long term.

\section{Cost Comparison of Induction Agents}

Selection of an induction agent is based on a variety of factors including risk of rejection due to donor or recipient, formulary of transplant center, or study protocol. Based on AWP, alemtuzumab is the least costly to institutions as it is provided to centers at no charge, followed by basiliximab and rATG, respectively. An economic and quality of life assessment of basiliximab versus rATG concluded that basiliximab demonstrated lower first year posttreatment costs with no differences seen in quality-adjusted survival. These same findings were also suggested in high-risk patients reporting total treatment costs lower for renal transplant recipients treated with basiliximab than those with rATG. No significant differences were reported between the groups in patient-assessed healthrelated quality of life within 1 year of transplant [19].

\section{Oral Maintenance Immunosuppression}

\section{Calcineurin Inhibitors}

Over the past two decades, CNIs have become the backbone and standard of care in immunosuppression regimens for prevention of acute rejection after solid organ transplantation. Cyclosporine (Sandimmune ${ }^{\circledR}$, Neoral ${ }^{\circledR}$ ) was the first CNI approved in 1983, followed by tacrolimus (Prograf ${ }^{\circledR}$ ) in 1994. Tacrolimus has largely replaced cyclosporine in terms of immunosuppressive therapy. Both exert their effects by binding their respective immunophilins (cyclophilin for cyclosporine and FK binding proteins for tacrolimus, respectively) to form a complex that inhibits the phosphatase, calcineurin, ultimately blocking transcription of interleukin-2, a T lymphocyte growth and survival factor [20]. A recent literature review of 12 studies comparing CNIs cyclosporine with tacrolimus in renal transplantation concluded that tacrolimus was the most cost-effective choice [21]. Cost savings were primarily due to lower rates of hospitalization as a result of fewer acute rejection episodes associated with tacrolimus. In an alternative analysis from the UK, patients maintained on tacrolimus had better short- and long-term outcomes, though long-term costs were found to be higher for those in the tacrolimus group. These authors reported higher initial 1-year costs in the tacrolimus group; however, for years 2-4 posttransplant, an average annual cost per patient was lower in the tacrolimus group due to a higher number of failed grafts in the cyclosporine group. The authors surmised that the benefit in terms of superior overall survival, survival with a functioning graft, and rejection-free survival makes tacrolimus a cost-effective alternative to cyclosporine [22].

\section{Anti-metabolites}

Mycophenolate mofetil (MMF; CellCept $\left.{ }^{\circledR}\right)$, in its active form, is an inhibitor of inosine-5'-monophosphate dehydrogenase (IMPDH), a key enzyme in the de novo pathway of purine (guanidine) nucleotide synthesis, inhibiting $\mathrm{T}$ and $\mathrm{B}$ lymphocytes that are dependent on this pathway from proliferating. Compared to azathioprine, the anti-metabolite used for decades to prevent transplant rejection, MMF is more effective, reducing acute rejection rates within the first 6 months posttransplant with a more favorable side effect profile [23]. A pharmacoeconomic review of studies in solid organ transplant recipients conducted within and outside the USA showed a cost benefit for MMF in the short term, postulating similar outcomes likely long term. These factors have offset the higher drug acquisition cost of MMF so that short-term costs are equivalent or cost saving when compared to azathioprine [24]. Most studies associate MMF with lower treatment costs and associated hospital admissions due to reduced incidence and severity of rejection episodes. Similarly, they take into account a lower cost due to lower incidence of graft failure with MMF.

While this may be true in kidney transplantation, liver transplantation has demonstrated different results. A yearly $\$ 4800$ per patient was estimated to be saved when using azathioprine compared to MMF at standard doses in liver transplant recipients [25]. The data are less convincing in terms of treatment efficacy and rejection avoidance. Other issues include the ongoing common gastrointestinal (GI) toxicities that limit dosing of MMF. Enteric-coated mycophenolate sodium (MPA; Myfortic ${ }^{\circledR}$ ) was developed and introduced as an alternative for use in patients unable to tolerate MMF due to GI side effects. Here, MPA was associated with a trend in improvements in the presence and severity of gastrointestinal symptoms, although statistical significance was not reached [26].

\section{Mammalian Target of Rapamycin Inhibitors}

Sirolimus (Rapamune ${ }^{\circledR}$ ) and everolimus (Zortress $\left.{ }^{\circledR}\right)$, both inhibitors of the activation of mammalian target of rapamycin (mTORi), have been studied in the transplant community as adjunct therapy to a CNI-based regimen as well as replacement for either a CNI or anti-metabolite. As the most recent approved oral agents, these agents tend to be more costly to both transplant centers and patients due to their branded status. Currently, only sirolimus tablets are available in a generic form. Favorable cost data generally results as the calculated or presumed cost avoided due to a CNI regimen adverse effect, namely, nephrotoxicity [27, 28]. In a study of cost-effectiveness of maintenance therapy with CNI versus those with CNI withdrawal, a survey of kidney transplant recipient data 
from 1990 to 2006 was entered into a lifetime Markov model. Here, the sirolimus-treated group showed increased patient survival, reduced graft loss, and therefore, increased quality-adjusted life-years (QALY) [27]. Cost savings was attributed to long-term benefits with sirolimus through improved renal function. However, in spite of the cost savings, this strategy has not been adapted uniformly in kidney transplantation due to a higher risk of acute rejection in the first year of transplantation and significant dyslipidemia albeit improved blood pressure [29, 30].

Another study evaluating pharmacoeconomic considerations for everolimus when compared to MMF suggests that everolimus is likely to be cost-neutral in clinical practice relative to established agents when evaluating direct health costs [28]. In this prospective study of nearly 600 recipients of deceased-donor kidney transplants on cyclosporine that were randomized to either everolimus $1.5 \mathrm{mg}$, everolimus $3 \mathrm{mg}$, or MMF, the mean overall costs were similar between study groups. Mean costs of cyclosporine were significantly lower in the everolimus groups (versus mycophenolate) likely due to synergistic effect seen with combination of everolimus and cyclosporine which facilitated reduction in cyclosporine dose. There were no major differences in efficacy between groups; there were fewer graft losses in the 1.5-mg everolimus group.

\section{Injectable/Biologics}

This class of immunosuppression shows the most promise, but as demonstrated below, the most cost. Indeed, 50 years ago, cost was not a consideration when choosing an agent, because then 1-year graft survival was so poor. The recognition that long-term survival is at a mean of 10 years and the recognition of complications of antibody-mediated injury for example are part of the equation in terms of choosing maintenance immunosuppressive therapy.

\section{Belatacept}

Belatacept (Nulojix ${ }^{\circledR}$ ), approved in June of 2011, is indicated for the prophylaxis of organ rejection in adult patients receiving a renal transplant. A soluble fusion protein, it binds to CD80 and CD86 on antigen-presenting cells inhibiting CD28-mediated co-stimulation of T lymphocytes [31]. Belatacept was introduced as a possible alternative to CNIs, and early studies showed less nephrotoxicity and cardiovascular side effects despite an early increased rate of acute rejection [32, 33]. Another advantage reported of belatacept is adherence tracking due to administration requirements as an intravenous infusion requiring administration in an outpatient clinic or hospital. Although belatacept is costly based on AWP, this increased cost might be acceptable in comparison to the high cost of caring for patients posttransplant. Recently, Caccippoli et al. used a 3-year Markov model to compare belatacept-based regimen versus a tacrolimus-based regimen in 954 adult renal (low to moderate risk) transplant recipients. From a Medicare perspective, 3-year costs were $\$ 147,876.00$ per patient in the belatacept group and $\$ 106,803.00$ per patient in the tacrolimus group [34]. The incremental costeffectiveness ratio (ICER) was $\$ 488,964$ per death averted. An alternative model found an ICER of \$1.07 million per death averted. Using AWP, the average cost per dose for a $70-\mathrm{kg}$ patient ranges from $\$ 2000.00$ to $\$ 3000.00$ depending on if the patient is in the induction or maintenance phase dosing interval. Other considerations include cost of nursing or administration, as belatacept is administered as an infusion requiring patients to be seen in a hospital or outpatient clinic. However, if improvement in toxicities of CNI could be demonstrated over time, then the high initial cost of this drug could be justifiable.

\section{Eculizumab}

While not currently indicated for use in solid organ transplantation, eculizumab (Soliris ${ }^{\circledR}$ ), a monoclonal antibody, acts by binding to complement protein $\mathrm{C} 5$, inhibiting its cleavage to $\mathrm{C} 5 \mathrm{a}$ and $\mathrm{C} 5 \mathrm{~b}$, preventing the generation of terminal complement complex C5b-9 [35]. The use in solid organ transplantation is off label and includes prevention and treatment of antibody-mediated rejection. Phase II studies are currently underway. This agent is also under investigation to mitigate delayed graft function as complement activation has been implicated in reperfusion injury (reviewed in [36••]). Cost considerations include significant and possibly long-term costs to centers and payers [37]. Cost-effectiveness might only be evident if a comparison with long-term cost of renal replacement therapy was conducted. An average dose, based on AWP, can range from $\$ 21,000.00$ to $\$ 29,000.00$ (900 and $1200 \mathrm{mg}$, respectively), with a slightly lower price based on eligible hospital 340B pricing or when dosed as an outpatient.

\section{Immune Globulin}

Immune globulin is commercially prepared preparations of the IgG fraction of antibodies derived from pooled human plasma. Although the exact mechanism by which it exerts immunomodulatory effects is not entirely clear [38], information has been published regarding immune globulin and its place in transplant therapy [39, 40]. Success has been documented as monotherapy or in combination with other agents in the highly sensitized patient, both improving transplant rates by reducing anti-HLA antibodies and as an immunomodulatory agent for those awaiting transplantation. Immune globulin is also frequently a component of desensitization protocols prior to transplantation. Intravenous immune globulin (IVIG) 
has been used in the treatment of antibody-mediated rejection along with infectious or immunodeficiency conditions in solid organ transplant patients. Cost information may be specific to practice site depending upon the formulation used; however, when one institution compared their average cost of an IVIG treatment containing regimen and the annual cost incurred by Medicare for care of ESRD patients and renal transplant recipients, the cost associated with the antibody-lowering protocol was substantially less than maintaining patients on dialysis for 4-5 years [39]. For an average dose for a $70-\mathrm{kg}$ patient (1-2 $\mathrm{g} / \mathrm{kg} / \mathrm{dose})$, the cost is $\$ 10,500-20,000$ per 1 - and 2-g dose, respectively, based on AWP. Some center-specific protocols require multiple courses of $1-2 \mathrm{~g}$ depending on the level of donor antibody detected and/or based on timing after transplantation. Thus, in conventional use, the therapy may be applied repeatedly as the above cost.

\section{Rituximab}

While not a standard member of the immunosuppressive drug regimen, the use of Rituximab (Rituxan ${ }^{\circledR}$ ), a monoclonal antibody against CD20, has been an adjunct in the armamentarium against antibody-mediated injury [41]. While the efficacy of this therapy in these indications is under study, transplant practitioners continue to use this medication off label for desensitization of recipient against donor HLA, for treatment of rejection, and in prevention of rejection [42] Dosing is based on body surface area and can be costly. For example, a single dose of $400 \mathrm{mg}$, based on AWP, would cost \$3384.

\section{Other Considerations in Transplantation}

\section{Impact of Generic Formulations}

Currently, many of the maintenance oral immunosuppressants are available in generic form including cyclosporine (2000), MMF (2008), tacrolimus (2009), mycophenolate sodium (2014), and sirolimus (2014). After the use of generic cyclosporine and issues that followed for many in the conversion, safety in using generic immunosuppression continues to be a consideration. Approval of generic therapies follows a different route for the FDA. Ensor et al. provides an excellent overview of this approval process [6••]. Here, they concluded that generics are a reasonable option for transplant recipients, but caution should be taken and education provided at the prescriber and patient level. Although unit price of medication may be less for generic formulations of medication, a concern remains for increased initial cost when conversion from brand product to generic due to cost resulted from increased laboratory monitoring and clinic visits. Other costs to consider include adverse effects, rejection episodes, etc. Immunosuppression was reported to account for approximately $6.6 \%$ of the total cost of renal transplantation in the first year, and when considering 2010 AWP, cyclosporine microemulsion, tacrolimus and mycophenolate generics are 15, 7, and $29 \%$ less expensive, respectively, than the branded products [6•*].

\section{The Cost of Non-adherence}

A recent descriptive survey of transplant centers captured the prevalence of immunosuppressive medication-related problems that kidney transplant recipients experience. When asked the question what percentage of kidney transplant recipients followed at a transplant center were not taking their maintenance immunosuppressive drugs as prescribed because of difficulties associated with their ability to pay for their medications, $28 \%$ of adult programs listed $0-5 \%$ of their patients not taking their immunosuppressive drugs as prescribed, $25.3 \%$ of centers reported problems in 6-10\% of patients, and $32 \%$ of programs listed $11-20 \%$ of patients had difficulty paying for medications. In adults, Medicare was ranked highest as the source of coverage for maintenance IS (68.5\%) with Medicaid $(29.8 \%)$ and private insurance (29\%) second and third, respectively [5]. Information gained from the U.S. Renal Data System reports in the year a kidney transplant recipient's graft fails, third-party payers experience an average annual expense of $\$ 82,765.00$. If the patient returns to dialysis, the average annual expense is $\$ 70,581.00$, and if the patient is retransplanted, the average cost is $\$ 106,373.00$. However, annual third-party reimbursements for a patient who has a functioning kidney transplant average $\$ 16,844.00$, making transplantation the most economically desirable long-term option [43].

\section{The Cost of Treating Allograft Rejection}

Newer agents have resulted in significantly fewer acute rejection episodes in the first year after transplantation when compared to older generation immunosuppression [44]. With the disparity in donor organs and longer waiting lists for transplant, transplant centers continue to take on more risk in terms of higher immune and graft functional risks (e.g., use of expanded criteria donors (ECD), desensitization protocols). Hospitalization and treatment with lymphocyte-depleting agents can be both costly to centers and patients. Newer agents used alone or in combination to treat antibody-mediated rejection can be even more costly. A recent study evaluating the economic impact of acute rejection analyzed Medicareinsured kidney transplant recipients and reported that among standard criteria donor (SCD) kidney recipients, antibodytreated acute rejection resulted in incremental marginal costs of $\$ 22,407$ in the first year, $\$ 18,603.00$ in the second year, and $\$ 13,909.00$ in the third year after transplantation [45••]. The increased marginal costs associated with non-antibody-treated acute rejection in the periods of evaluation were lower at $\$ 14$, 
122.00 in the first year, $\$ 7852.00$ in the second year, and $\$ 8234.00$ in the third year after SCD transplantation. Patterns were similar among living donor and ECD transplant recipients with a few exceptions. Reported proportion of total costs attributable to AR accounted for 2.3 to $3.8 \%$ of total period costs among SCD and LD recipients, and a similar proportion of year 1 and 2 period costs among ECD recipients.

\section{The 340B Drug Pricing Program}

Established in 1992, the 340B prime vendor program was established to provide financial relief to specific organizations that provide medical care to the underserved. The purpose of this federally enacted in law program was to reduce the price of outpatient prescription and over-the-counter drugs to medical centers that are federally qualified health centers and hospitals. Participating hospitals in 340B must follow strict policies and procedures to ensure adherence to program eligibility guidelines. The Program establishes a ceiling price, which is the maximum price a manufacturer can charge. Those prices are calculated quarterly by manufacturers and can result in 20 to $50 \%$ of the AWP for entities enrolled in this program [46]. Although meeting eligibility criteria, remaining compliant and preparing for government audits poses challenges and may actually increase initial costs for institutions, but does provide significant long-term cost savings when medications are used and dispensed on the outpatient level. While no study has been undertaken regarding the impact of this program nationwide on immunosuppression, a recent retrospective analysis of its impact in two community health centers for 2772 covered individuals demonstrated an average cost savings of $\$ 62$ [47]. While seemingly small savings, this was for more common medical conditions and certainly holds specific impact on centers serving patient populations that lack medication coverage.

\section{Cost of New Drug Development}

DiMasi et al. estimated an average price of $\$ 802$ million (in year 2000 dollars) for the discovery and development of a new chemical entity or essentially a new drug. This cost considers cost of ailed products along with the cost of those entities that receive approval [48]. Dickson et al. reported that there has been an increase in the average time it takes for drug approval ( 7.9 years in 1960 s to 12.8 years in 1990 s), due in part to increased length of clinical trials, which authors believe contribute to the increase in cost of development [49], and the ongoing issues of defining endpoints, particularly in transplantation, have led to significant barriers in new drug development. Some cost may be reduced here if primary development is for another disease and the drug repurposed, but the impact of this financially remains uncertain.

\section{The Affordable Care Act}

The impact of the Affordable Care Act in solid organ transplantation remains another unknown in the understanding of immunosuppression cost [50]. Medicare coverage is limited, providing for the procedure but only $80 \%$ of the cost of immunosuppressive medications for 36 months after transplantation and no coverage thereafter unless disabled or age eligible. This gap in cost, estimated to be $\sim \$ 25,000$ per year, is for standard medications [51]. It is theoretically possible that the ACA's insurance exchanges will include lifetime coverage for immunosuppressive drugs. The exact coverage that will be provided and its implementation including lifetime coverage offered at perhaps a lower price option are yet to be determined. The cost savings of such lifetime coverage may be related due to reduced non-adherence [5, 52], thus minimizing immune-mediated injuries that contribute to late allograft failure.

\section{Conclusion}

The cost of kidney transplantation is clearly affected by the need for lifetime immunosuppressive drug use. As transplant recipients' life span and long-term allograft survivals improve, this cost will remain a fairly constant financial requirement. As complications develop or more immune risk transplants are undertaken, the added expense of the biologics comes into play. There is ongoing demand, too, for therapies with less long-term toxicities that contribute to cardiac morbidity, and these newer agents are costly in terms of their use. Offsetting this concern is the possibility of better graft function, less graft immune injury episodes, and better adherence. While data are limited, the lack of affordability for the uninsured of conventional therapy argues for improved support and access for chronic immunosuppression.

Acknowledgments RBM is supported in part by U01AI084150, U19A1070119, and R01DK083390 and funding from the University of Alabama at Birmingham.

\section{Compliance with Ethics Guidelines}

Conflict of Interest Alexandra James declares that she has no conflict of interest.

Roslyn B. Mannon reports grants and other (consultant, DSMB member) from Astellas and grants from Amgen, BMS, and Alexion.

Human and Animal Rights and Informed Consent This article does not contain any studies with human or animal subjects performed by any of the authors. 


\section{References}

Papers of particular interest, published recently, have been highlighted as:

-• Of major importance

1. Bentley TS, Hanson SG. 2011 US organ and tissue transplant cost estimates and discussion. WI: Brookfield; 2011.

2. Loubeau PR, Loubeau JM, Jantzen R. The economics of kidney transplantation versus hemodialysis. Prog Transplant. 2001;11(4): 291-7.

3. Kasiske BL, Cohen D, Lucey MR, Neylan JF. Payment for immunosuppression after organ transplantation. American Society of Transplantation. JAMA. 2000;283(18):2445-50.

4. Gordon EJ, Prohaska TR, Sehgal AR. The financial impact of immunosuppressant expenses on new kidney transplant recipients. Clin Transplant. 2008;22(6):738-48.

5. Evans RW, Applegate WH, Briscoe DM, Cohen DJ, Rorick CC, Murphy BT, et al. Cost-related immunosuppressive medication nonadherence among kidney transplant recipients. Clin J Am Soc Nephrol. 2010;5(12):2323-8.

6.• Ensor CR, Trofe-Clark J, Gabardi S, McDevitt-Potter LM, Shullo MA. Generic maintenance immunosuppression in solid organ transplant recipients. Pharmacotherapy. 2011;31(11):1111-29. Excellent analysis and discussion regarding generic immunosuppressive therapy and its cost benefits.

7. Novartis Pharmaceuticals. Simulect (Package Insert). 2005.

8. Webster AC, Ruster LP, McGee R, Matheson SL, Higgins GY, Willis NS, et al. Interleukin 2 receptor antagonists for kidney transplant recipients. Cochrane Database Syst Rev. 2010;1, CD003897.

9. Chapman TM, Keating GM. Basiliximab: a review of its use as induction therapy in renal transplantation. Drugs. 2003;63(24): 2803-35.

10. Sanofi Corporation. Thymoglobulin Package Insert. 2008.

11. Matas AJ, Smith JM, Skeans MA, Thompson B, Gustafson SK, Schnitzler MA, et al. OPTN/SRTR 2012 Annual Data Report: kidney. Am J Transplant. 2014;14 Suppl 1:11-44.

12. Schnitzler MA, Woodward RS, Lowell JA, Amir L, Schroeder TJ, Singer GG, et al. Economics of the antithymocyte globulins Thymoglobulin and Atgam in the treatment of acute renal transplant rejection. Pharmacoeconomics. 2000;17(3):287-93.

13. Miller JT, Collins CD, Stuckey LJ, Luan FL, Englesbe MJ, Magee $\mathrm{JC}$, et al. Clinical and economic outcomes of rabbit antithymocyte globulin induction in adults who received kidney transplants from living unrelated donors and received cyclosporine-based immunosuppression. Pharmacotherapy. 2009;29(10):1166-74.

14. Marfo K, Akalin E, Wang C, Lu A. Clinical and economic analysis of short-course versus standard-course antithymocyte globulin (rabbit) induction therapy in deceased-donor renal transplant recipients. Am J Health Syst Pharm. 2011;68(23):2276-82.

15. Peddi VR, Bryant M, Roy-Chaudhury P, Woodle ES, First MR. Safety, efficacy, and cost analysis of thymoglobulin induction therapy with intermittent dosing based on CD3+ lymphocyte counts in kidney and kidney-pancreas transplant recipients. Transplantation. 2002;73(9):1514-8

16. McGillicuddy JW, Taber DJ, Pilch NA, Kohout RK, Bratton CF, Chavin KD, et al. Clinical and economic analysis of delayed administration of antithymocyte globulin for induction therapy in kidney transplantation. Prog Transplant. 2013;23(1):33-8.

17. Sanofi Corporation. Campath package insert. 2009.

18.• Hanaway MJ, Woodle ES, Mulgaonkar S, Peddi VR, Kaufman DB, First MR, et al. Alemtuzumab induction in renal transplantation. N Engl J Med. 2011;364(20):1909-19. Landmark study comparing standard depletional induction using rabbit ATG versus Campath.

19. Polsky D, Weinfurt KP, Kaplan B, Kim J, Fastenau J, Schulman KA. An economic and quality-of-life assessment of basiliximab vs antithymocyte globulin immunoprophylaxis in renal transplantation. Nephrol Dial Transplant. 2001;16(5):1028-33.

20. DiPiro J, Yee G, Matzke G, Wells B, Posey L. Pharmacotherapy: a pathophysiologic approach. New York: McGraw-Hill; 2005.

21. Miners AH, Yao G, Raftery J, Taylor RS. Economic evaluations of calcineurin inhibitors in renal transplantation: a literature review. Pharmacoeconomics. 2007;25(11):935-47.

22. Orme ME, Jurewicz WA, Kumar N, McKechnie TL. The cost effectiveness of tacrolimus versus microemulsified cyclosporin: a 10year model of renal transplantation outcomes. Pharmacoeconomics. 2003;21(17):1263-76.

23. Nakazawa S, Kishikawa H, Kawamura M, Ueda N, Hirai T, Nishimura K. Conversion to mycophenolate mofetil from azathioprine shows significant positive effect on graft function in longterm past-kidney transplantation stable-state patients. Transplant Proc. 2014;46(2):411-4.

24. Young M, Plosker GL. Mycophenolate mofetil: a pharmacoeconomic review of its use in solid organ transplantation. Pharmacoeconomics. 2002;20(10):675-713.

25. Germani G, Pleguezuelo M, Villamil F, Vaghjiani S, Tsochatzis E, Andreana L, et al. Azathioprine in liver transplantation: a reevaluation of its use and a comparison with mycophenolate mofetil. Am J Transplant. 2009;9(8):1725-31.

26. Langone AJ, Chan L, Bolin P, Cooper M. Enteric-coated mycophenolate sodium versus mycophenolate mofetil in renal transplant recipients experiencing gastrointestinal intolerance: a multicenter, double-blind, randomized study. Transplantation. 2011;91(4):470-8.

27. Earnshaw SR, Graham CN, Irish WD, Sato R, Schnitzler MA. Lifetime cost-effectiveness of calcineurin inhibitor withdrawal after de novo renal transplantation. J Am Soc Nephrol. 2008;19(9): 1807-16.

28. Holmes M, Chilcott J, Walters S, Whitby S, Akehurst R. Economic evaluation of everolimus versus mycophenolate mofetil in combination with cyclosporine and prednisolone in de novo renal transplant recipients. Transpl Int. 2004;17(4):182-7.

29. Johnson RW, Kreis H, Oberbauer R, Brattstrom C, Claesson K, Eris J. Sirolimus allows early cyclosporine withdrawal in renal transplantation resulting in improved renal function and lower blood pressure. Transplantation. 2001;72(5):777-86.

30. Oberbauer R, Kreis H, Johnson RW, Mota A, Claesson K, Ruiz JC, et al. Long-term improvement in renal function with sirolimus after early cyclosporine withdrawal in renal transplant recipients: 2-year results of the Rapamune Maintenance Regimen Study. Transplantation. 2003;76(2):364-70.

31. Bristol-Myers Squibb. Nulojix Package Insert. 2014.

32. Durrbach A, Pestana JM, Pearson T, Vincenti F, Garcia VD, Campistol J, et al. A phase III study of belatacept versus cyclosporine in kidney transplants from extended criteria donors (BENEFITEXT study). Am J Transplant. 2010;10(3):547-57.

33. Vincenti F, Charpentier B, Vanrenterghem Y, Rostaing L, Bresnahan B, Darji P, et al. A phase III study of belatacept-based immunosuppression regimens versus cyclosporine in renal transplant recipients (BENEFIT study). Am J Transplant. 2010;10(3): 535-46.

34. Caccioppoli V, MacLasco A, Chan K, Gandhi R, LaBella S, Somers $\mathrm{L}$, et al. Cost effectiveness of a belatacept-based regimen in kidney transplant recipients. American Transplant Congress: American Journal of Transplantation; 2013. p. 183

35. Alexion Pharmaceuticals. Soliris Package Insert. 2014.

36.• Schroppel B, Legendre C. Delayed kidney graft function: from mechanism to translation. Kidney Int. 2014;86:251-58. 
Comprehensive review on delayed graft injury and potential pathways to mitigate injury.

37. Barnett AN, Asgari E, Chowdhury P, Sacks SH, Dorling A, Mamode N. The use of eculizumab in renal transplantation. Clin Transplant. 2013;27(3):E216-29.

38. Boros P, Gondolesi G, Bromberg JS. High dose intravenous immunoglobulin treatment: mechanisms of action.

39. Jordan S, Cunningham-Rundles C, McEwan R. Utility of intravenous immune globulin in kidney transplantation: efficacy, safety, and cost implications. Am J Transplant. 2003;3(6):653-64.

40. Jordan SC, Toyoda M, Kahwaji J, Vo AA. Clinical aspects of intravenous immunoglobulin use in solid organ transplant recipients. Am J Transplant. 2011;11(2):196-202.

41. Barnett ANR, Hadjianastassiou VG, Mamode N. Rituximab in renal transplantation.

42. Pescovitz MD. Rituximab, an anti-cd20 monoclonal antibody: history and mechanism of action. Am J Transplant. 2006;6(5 Pt 1): 859-66.

43. National Institutes of Health NIDDK. Renal Data System: USRDS 2009 Annual Data Report: atlas of chronic kidney disease and endstage renal disease in the United States. Bethesda, MD: 2009.

44. Lodhi SA, Lamb KE, Meier-Kriesche HU. Solid organ allograft survival improvement in the United States: the long-term does not mirror the dramatic short-term success. Am J Transplant. 2011;11(6):1226-35.

45.• Gheorghian A, Schnitzler MA, Axelrod DA, Kalsekar A, L'Italien $\mathrm{G}$, Lentine KL. The implications of acute rejection and reduced allograft function on health care expenditures in contemporary US kidney transplantation. Transplantation. 2012;94(3):241-9. Critical analysis of US data of the cost of acute rejection and allograft dysfunction following kidney transplantation.

46. Werling K, Abraham S, Strelec J. The 340B Drug Pricing Program: an opportunity for savings, if covered entities such as disproportionate share hospitals and federally qualified health centers know how to interpret the regulations. J Health Care Finance. 2007;34(2): 57-70.

47. Castellon YM, Bazargan-Hejazi S, Masatsugu M, Contreras R. The impact of patient assistance programs and the 340B Drug Pricing Program on medication cost. Am J Manag Care. 2014;20(2):14650.

48. DiMasi JA, Hansen RW, Grabowski HG. The price of innovation: new estimates of drug development costs. J Health Econ. 2003;22(2):151-85.

49. Dickson M, Gagnon JP. The cost of new drug discovery and development. Discov Med. 2004;4(22):172-9.

50. Rizk CS, Singh SN. Implications of the Affordable Care Act for kidney transplantation. Virtual Mentor. 2012;14(3):250-5.

51. Cohen DJ, Murphy B. Drug coverage for transplantation turns into political football: big business trumps patients. Clin J Am Soc Nephrol. 2010;5(5):746-7.

52. Rettig RA. The social contract and the treatment of permanent kidney failure. JAMA. 1996;275(14):1123-6. 